\title{
Discrete coherent and squeezed states of many-qudit systems
}

\author{
Andrei B. Klimov, ${ }^{1}$ Carlos Muñoz, ${ }^{1}$ and Luis L. Sánchez-Soto ${ }^{2}$ \\ ${ }^{1}$ Departamento de Física, Universidad de Guadalajara, 44420 Guadalajara, Jalisco, Mexico \\ ${ }^{2}$ Departamento de Óptica, Facultad de Física, Universidad Complutense, 28040 Madrid, Spain
}

(Dated: November 11, 2018)

\begin{abstract}
We consider the phase space for a system of $n$ identical qudits (each one of dimension $d$, with $d$ a primer number) as a grid of $d^{n} \times d^{n}$ points and use the finite field $\operatorname{GF}\left(d^{n}\right)$ to label the corresponding axes. The associated displacement operators permit to define $s$-parametrized quasidistribution functions in this grid, with properties analogous to their continuous counterparts. These displacements allow also for the construction of finite coherent states, once a fiducial state is fixed. We take this reference as one eigenstate of the discrete Fourier transform and study the factorization properties of the resulting coherent states. We extend these ideas to include discrete squeezed states, and show their intriguing relation with entangled states between different qudits.
\end{abstract}

PACS numbers: 03.65.Ta,03.65.Fd,42.50.Dv

\section{INTRODUCTION}

The concept of phase-space representation of quantum mechanics, introduced in the pioneering works of Weyl [1], Wigner [2], and Moyal [3], is a very useful and enlightening approach that sheds light on the correspondence between quantum and classical worlds.

Numerous applications of the phase-space methods to physical problems have been extensively discussed in the last decades [4, 5, 6, 7]. However, much of this subject is usually illustrated in terms of continuous variables, most often position and momentum.

For discrete systems, or qudits in the modern parlance of quantum information, things are less straightforward. Since the dynamical symmetry group for a qudit is $\mathrm{SU}(d)$, one may be tempted to interpret its associated phase space as a generalized Bloch sphere [8, 9], which is supported by the rigorous construction of Kostant [10] and Kirilov [11] in terms of coadjoint orbits. Even if this picture is quite popular, especially when applied to qubits, one can rightly argue that there is a lot of information redundancy there and that the phase space should be a grid of points, as one could expect for a truly discrete system.

Indeed, apart from some noteworthy exceptions $[12,13$, 14], nowadays there is a wide consensus in picturing the phase space for a qudit as a $d \times d$ grid. This can be traced back to the elegant approach proposed by Schwinger [15, 16, 17], who clearly recognized that the expansion of arbitrary operators in terms of certain operator basis was the crucial mathematical concept in setting such a grid. These ideas have been rediscovered and developed further by several authors [18, 19, 20, 21, 22, 23, 24], although the contributions of Wootters [25, 26, 27, 28] and Galetti and coworkers [29, 30, 31] are worth stressing.

To equip this grid with properties analogous to the geometry of an ordinary plane, it turns out essential [32, 33, 34] to label the axes in terms of the elements of a finite field $\mathrm{GF}(d)$ with $d$ elements. It is well known that such a field exist only when the dimension is a prime or a power of a prime [35]. This, of course, gives a special role to qudits in prime dimen- sions, but also is ideally suited to deal with systems of $n$ of these qudits.

Once the natural arena is properly established, the next question is how to represent states (and operators) on phase space. This is done through quasidistribution functions, which allow for the calculation of quantum averages in a way that exactly parallels classical mechanics. There are, however, important differences with respect to a classical description: they come from the noncommuting nature of conjugate quantities (like position and momentum), which precludes their simultaneous precise measurement and, therefore, imposes a fundamental limit on the accuracy with which we can determine a point in phase space. As a distinctive consequence of this, there is no unique rule by which we can associate a classical phase-space variable to a quantum operator. Therefore, depending on the operator ordering, various quasidistributions can be defined. For continuous variables, the best known are the Glauber-Sudarshan $P$ function [36, 37], the Husimi $Q$ function [38], and the Wigner $W$ function [39], corresponding to normal, antinormal, and symmetric order, respectively, in the associated characteristic functions. In fact, all of them are special cases of the $s$-parametrized quasidistributions introduced by Cahill and Glauber [40].

The problem of generalizing these quasidistributions (mainly the Wigner function) to finite systems has also a long history. Much of the previous literature has focused on spin variables, trying to represent spin states by continuous functions of angle variables. This idea was initiated by Stratonovich [41], Berezin [42], and Agarwal [43]. The resulting Wigner function, naturally related to the SU(2) dynamical group, has been further studied by a number of authors [44, 45, 46, 47], has been applied to some problems in quantum optics [48, 49] and extended to more general groups [50].

However, these Wigner functions are not defined in a discrete phase space. A detailed review of possible solutions can be found in Ref. [51]. Perhaps, the most popular one is due to Wootters and coworkers [52, 53, 54], which imposes a structure by assigning a quantum state to each line in phase space. Any good assignment of quantum states to lines is called a "quantum net" and can be used to define a discrete Wigner 
function. In this paper, we show how to introduce a set of $s$ parametrized functions, in close correspondence with the continuous case. We emphasize that, although some interesting work has been done in this direction by using a mod $d$ invariance [55, 56], our approach works quite well for many-qudit systems.

Another essential ingredient in any phase-space description is the notion of coherent states [57]. This is firmly established for continuous variables and can easily extended for other dynamical symmetry groups [58]. However, for discrete systems we have again a big gap waiting to be filled. The reason for this can be traced back to the fact that, as brightly pointed out in Ref. [59], in the continuum we have one, and only one, harmonic oscillator, while in the discrete there are a lot of candidates for that role, each one surely with its virtues, but surely no undisputed champion.

The strategy we adopt to deal with this problem is to look for eigenstates of the discrete Fourier transform [60]. For continuous variables, they have a very distinguishable behavior that is at the basis of the remarkable properties of coherent states. We explore this approach, getting a strikingly simple family of discrete coherent states (even for many qudits) fulfilling all the requirements.

To put the icing on the cake, we also extend the notion of squeezed states for these systems [61]. For a single qudit, the resulting states have nice and expected properties. However, when they really appear as highly interesting is for multipartite systems, since they present an intriguing relation with entanglement.

In short, the program developed in this paper can be seen as a handy toolbox for the phase-space analysis of many-qudit systems, which should be of interest to a large interdisciplinary community working in these topics.

\section{PHASE SPACE FOR CONTINUOUS VARIABLES}

In this Section we briefly recall the relevant structures needed to set up a phase-space description of Cartesian quantum mechanics. This will facilitate comparison with the discrete case later on. For simplicity, we choose one degree of freedom only, so the associated phase space is the plane $\mathbb{R}^{2}$.

The relevant observables are the Hermitian coordinate and momentum operators $\hat{q}$ and $\hat{p}$, with canonical commutation relation (with $\hbar=1$ throughout)

$$
[\hat{q}, \hat{p}]=i \hat{\mathbb{1}}
$$

so that they are the generators of the Heisenberg-Weyl algebra. Ubiquitous and profound, this algebra has become the hallmark of noncommutativity in quantum theory. To avoid technical problems with the unbounded operators $\hat{q}$ and $\hat{p}$, it is convenient to work with their unitary counterparts [62]

$$
\hat{U}(q)=\exp (-i q \hat{p}), \quad \hat{V}(p)=\exp (i p \hat{q}),
$$

which generate translations in position and momentum, respectively. The commutation relations are then expressed in the Weyl form

$$
\hat{V}(p) \hat{U}(q)=e^{i q p} \hat{U}(q) \hat{V}(p)
$$

Their infinitesimal form immediately gives (2.1), but 2.3) is more useful in many instances.

In terms of $\hat{U}$ and $\hat{V}$ a displacement operator can be introduced as

$$
\hat{D}(q, p)=e^{-i q p / 2} \hat{U}(p) \hat{V}(q)
$$

which usually is presented in the entangled form $\hat{D}(q, p)=$ $\exp [i(p \hat{q}-q \hat{p})]$. However, this cannot be done in more general situations.

The $\hat{D}(q, p)$ form a complete orthonormal set (in the trace sense) in the space of operators acting on $\mathcal{H}$ (the Hilbert space of square integrable functions on $\mathbb{R}$ ). The unitarity imposes that $\hat{D}^{\dagger}(q, p)=\hat{D}(-q,-p)$, and $\hat{D}(0,0)=\hat{\mathbb{1}}$. In addition, they obey the simple composition law

$$
\hat{D}\left(\hat{q}_{1}, \hat{p}_{1}\right) \hat{D}\left(\hat{q}_{2}, \hat{p}_{2}\right)=e^{i\left(p_{1} q_{2}-q_{1} p_{2}\right) / 2} \hat{D}\left(\hat{q}_{1}+\hat{q}_{2}, \hat{q}_{2}+\hat{p}_{2}\right) \text {. }
$$

The displacement operators constitute a basic element for the notion of coherent states. Indeed, if we choose a fixed normalized reference state $\left|\psi_{0}\right\rangle$, we can define these coherent states as [58]

$$
|q, p\rangle=\hat{D}(q, p)\left|\psi_{0}\right\rangle
$$

so they are parametrized by phase-space points. These states have a number of remarkable properties, inherited from those of $\hat{D}(q, p)$. In particular, $\hat{D}(q, p)$ transforms any coherent state in another coherent state:

$$
\hat{D}\left(\hat{q}_{1}, \hat{p}_{1}\right)\left|q_{2}, p_{2}\right\rangle=e^{i\left(p_{1} q_{2}-q_{1} p_{2}\right) / 2}\left|q_{1}+q_{2}, q_{2}+p_{2}\right\rangle .
$$

We need to determine the fiducial vector $\left|\psi_{0}\right\rangle$. The standard choice is to take it as the vacuum $|0\rangle$. This has quite a number of relevant properties, but the one we want to stress for what follows is that $|0\rangle$ is an eigenstate of the Fourier transform (as they are all the Fock states) [63], and so is the Gaussian

$$
\psi_{0}(q)=\langle q \mid 0\rangle=\frac{1}{\pi^{1 / 4}} \exp \left(-q^{2} / 2\right)
$$

in appropriate units. In addition, this wavefunction represents a minimum uncertainty state, namely

$$
(\Delta q)^{2}(\Delta p)^{2}=\frac{1}{4}
$$

where $(\Delta q)^{2}$ and $(\Delta p)^{2}$ are the corresponding variances.

Our next task is to map the density matrix $\hat{\varrho}$ into a function defined on $\mathbb{R}^{2}$. There exists a whole class of these quasidistribution functions, related to different orderings of $\hat{q}$ and $\hat{p}$. The corresponding mappings are generated by an $s$-ordered kernel

$$
W_{\hat{\varrho}}^{(s)}(q, p)=\operatorname{Tr}\left[\varrho \hat{w}^{(s)}(q, p)\right],
$$


where $\hat{w}^{(s)}$ is the double Fourier transform of the displacement operator with a weight fixed by the operator ordering

$$
\begin{aligned}
\hat{w}^{(s)}(q, p) & =\frac{1}{(2 \pi)^{2}} \int_{\mathbb{R}^{2}} \exp \left[-i\left(p q^{\prime}-q p^{\prime}\right)\right] \hat{D}\left(q^{\prime}, p^{\prime}\right) \\
& \times\left\langle\psi_{0}\left|\hat{D}\left(q^{\prime}, p^{\prime}\right)\right| \psi_{0}\right\rangle^{-s} d q^{\prime} d p^{\prime}
\end{aligned}
$$

and $s \in[-1,1]$. The mapping is invertible, so that

$$
\hat{\varrho}=\frac{1}{(2 \pi)^{2}} \int_{\mathbb{R}^{2}} \hat{w}^{(-s)}(q, p) W^{(s)}(q, p) d q d p .
$$

The Hermitian kernels $\hat{w}^{(s)}(q, p)$ are also a complete traceorthonormal set and they transform properly under displacements

$$
\hat{w}^{(s)}(q, p)=\hat{D}(q, p) \hat{w}^{(s)}(0,0) \hat{D}^{\dagger}(q, p) .
$$

The symmetric ordering $(s=0)$ corresponds to the Wigner function $W(q, p)$ and the associated kernel $\hat{w}^{(0)}(0,0)$ is just $2 \hat{\mathcal{P}}$, where $\hat{\mathcal{P}}$ is the parity operator. For the antinormal ordering $(s=-1)$, which corresponds to the Husimi $Q$ function, $\hat{w}^{(0)}(0,0)=|0\rangle\langle 0| / \pi$.

The quasidistribution functions (2.10) fulfill all the basic properties required for any good probabilistic description. First, due to the Hermiticity of $\hat{w}^{(s)}(q, p)$, they are real for Hermitian operators. Second, on integrating $W^{(s)}(q, p)$ over one variable, the probability distribution of the conjugate variable is reproduced. And finally, $W^{(s)}(q, p)$ is covariant, which means that for the displaced state $\hat{\varrho}^{\prime}=$ $\hat{D}\left(q_{0}, p_{0}\right) \hat{\varrho} \hat{D}^{\dagger}\left(q_{0}, p_{0}\right)$, one has

$$
W_{\hat{\varrho}^{\prime}}^{(s)}(q, p)=W_{\hat{\varrho}}^{(s)}\left(q-q_{0}, p-p_{0}\right),
$$

so that these functions follow displacements rigidly without changing their form, reflecting the fact that physics should not depend on a certain choice of the origin.

\section{SINGLE QUDIT}

\section{A. Discrete phase space}

We consider a system living in a Hilbert space $\mathcal{H}_{d}$, whose dimension $d$ is assumed from now on to be a prime number. We choose a computational basis $|\ell\rangle$ in $\mathcal{H}_{d}(\ell=0, \ldots, d-$ 1) which we arbitrarily interpret as the "position" basis, with periodic boundary conditions $|\ell+d\rangle=|\ell\rangle$. The conjugate "momentum" basis can be introduced by means of the discrete Fourier transform [64], that is

$$
|\tilde{\ell}\rangle=\hat{\mathcal{F}}|\ell\rangle,
$$

where

$$
\hat{\mathcal{F}}=\frac{1}{\sqrt{d}} \sum_{\ell, \ell^{\prime}=0}^{d-1} \omega\left(\ell \ell^{\prime}\right)|\ell\rangle\left\langle\ell^{\prime}\right|,
$$

and we use the notation

$$
\omega(\ell)=\omega^{\ell}=\exp (i 2 \pi \ell / d),
$$

$\omega=\exp (i 2 \pi / d)$ being a $d$ th root of the unity. Whenever we do not specify the ranges in a sum, we understand the index running all its natural domain.

Once we have position and momentum basis, the phase space turns out to be a periodic $d \times d$ grid of points; i.e., the torus $\mathbb{Z}_{d} \times \mathbb{Z}_{d}$, where $\mathbb{Z}_{d}$ is the field of the integer numbers modulo $d$.

Mimicking the approach in Sec. [II, we introduce the operators $\hat{U}$ and $\hat{V}$, which generate finite translations in position and momentum, respectively. In fact, $\hat{U}$ generates cyclic shifts in the position basis, while $\hat{V}$ is diagonal

$$
\hat{U}^{n}|\ell\rangle=|\ell+n\rangle, \quad \hat{V}^{m}|\ell\rangle=\omega(m \ell)|\ell\rangle,
$$

where addition and multiplication must be understood modulo $d$. Conversely, $\hat{U}$ is diagonal in the momentum basis and $\hat{V}$ acts as a shift, which is reflected also by the fact that

$$
\hat{V}=\mathcal{F} \hat{U} \mathcal{F}^{\dagger},
$$

much in the spirit of the standard way of looking at complementary variables in the infinite-dimensional Hilbert space: the position and momentum eigenstates are Fourier transform one of the other. Note that the operators $\hat{U}$ and $\hat{V}$ are generalizations of the Pauli matrices $\sigma_{x}$ and $\sigma_{z}$, so many authors use the notation $\hat{X}$ and $\hat{Z}$ for them.

One can directly check the identity

$$
\hat{V}^{m} \hat{U}^{n}=\omega(m n) \hat{U}^{n} \hat{V}^{m},
$$

which is the finite-dimensional version of the Weyl form of the commutation relations and show that they obey a generalized Clifford algebra [65].

One may be tempted to define discrete position and momentum operators. A possible way to achieve this is to write [66]

$$
\hat{U}=\exp (-i 2 \pi \hat{P} / d), \quad \hat{V}=\exp (i 2 \pi \hat{Q} / d),
$$

with

$$
\hat{Q}=\sum_{\ell} \ell|\ell\rangle\left\langle\ell\left|, \quad \hat{P}=\sum_{\tilde{\ell}} \tilde{\ell}\right| \tilde{\ell}\right\rangle\langle\tilde{\ell}| .
$$

However, for finite quantum systems the Heisenberg-Weyl group is discrete, there is no Lie algebra (that is, there are no infinitesimal displacements) and the role of position and momentum is limited. For this reason our formalism is mainly based on the operators $\hat{U}$ and $\hat{V}$.

Next we introduce the displacement operators

$$
\hat{D}(m, n)=\phi(m, n) \hat{U}^{n} \hat{V}^{m},
$$

where $\phi(m, n)$ is a phase required to avoid plugging extra factors when acting with $\hat{D}$. The conditions of unitarity and periodicity restrict the possible values of $\phi$. One relevant choice (for $d>2$ ) that have been analyzed in the literature is [51]

$$
\phi(m, n)=\omega\left(2^{-1} m n\right),
$$


where $2^{-1}$ is the multiplicative inverse of 2 in $\mathbb{Z}_{d}$. For qubits, $\phi(m, n)$ may be taken as $\phi(m, n)= \pm i^{m n}$.

Without entering in technical details, this choice guarantees all the good properties, in particular the analogous to Eq. (2.5):

$$
\begin{gathered}
\hat{D}\left(m_{1}, n_{1}\right) \hat{D}\left(m_{2}, n_{2}\right)=\omega\left[2^{-1}\left(m_{1} n_{2}-m_{2} n_{1}\right)\right] \\
\times \hat{D}\left(m_{1}+m_{2}, n_{1}+n_{2}\right),
\end{gathered}
$$

and the following relation

$$
\frac{1}{d} \sum_{m, n} \hat{D}(m, n)=\hat{\mathcal{P}}
$$

where $\hat{\mathcal{P}}$ is the parity operator $\hat{\mathcal{P}}|\ell\rangle=|-\ell\rangle$ modulo $d$. Physically, this is the basis for translational covariance and this also means that $\hat{D}(m, n)$ translates the standard basis states cyclically $m$ places in one direction and $n$ places in the orthogonal one, as one would expect from a displacement operator.

\section{B. Coherent states}

Once a proper displacement operator has been settled, the coherent states for a single qudit can be defined as

$$
|m, n\rangle=\hat{D}(m, n)\left|\psi_{0}\right\rangle,
$$

where $\left|\psi_{0}\right\rangle$ is again a reference state. These states are also labeled by points of the discrete phase space, as it should be.

A possible choice [67,68] is to use for $\left|\psi_{0}\right\rangle$ the ground state of the Harper Hamiltonian [69]

$$
\hat{H}=2-\frac{\hat{U}+\hat{U}^{\dagger}}{2}-\frac{\hat{V}+\hat{V}^{\dagger}}{2},
$$

which is considered as the discrete counterpart of the harmonic oscillator with the proper periodicity conditions. While such a replacement is interesting, it is by no means unique.

We prefer to take a different route, pioneered by Galetti and coworkers [60]. We use again as a guide the analogy with the continuous case and look for eigenstates $|f\rangle$ of the discrete Fourier transform, which play the role of Fock states for our problem and are determined by

$$
\langle\ell|\hat{\mathcal{F}}| f\rangle=i^{\ell}\langle\ell \mid f\rangle .
$$

Obviously, the fact that $\hat{\mathcal{F}}^{4}=\hat{\mathbb{1}}$ implies that it has four eigenvalues: $1,-1, i$, and $-i$. The solutions of this equation were fully studied by Mehta [70] (see also Ruzzi [59]). Taking $\left|\psi_{0}\right\rangle$ as the "ground" state (i.e., $\ell=0$ ) one gets

$$
\left|\psi_{0}\right\rangle=\frac{1}{\sqrt{C}} \sum_{k \in \mathbb{Z}} \sum_{\ell} \omega(k \ell) e^{-\frac{\pi}{d} k^{2}}|\ell\rangle
$$

and the normalization constant $C$ is given by

$$
C=\sum_{k \in \mathbb{Z}} e^{-\frac{2 \pi}{d} k^{2}}=\vartheta_{3}\left(0 \mid e^{-\frac{2 \pi}{d}}\right)
$$

$\vartheta_{3}$ being the third Jacobi function [71]. Note in passing that this fiducial state can be alternatively represented as

$$
\left|\psi_{0}\right\rangle=\frac{1}{\sqrt{C}} \sum_{\ell} \vartheta_{3}\left(\frac{\pi \ell}{d} \mid e^{-\frac{\pi}{d}}\right)|\ell\rangle .
$$

The appearance of the Jacobi function in the present context can be directly understood by realizing that this function is a periodic eigenstate of the discrete Fourier operator with eigenvalue +1 and period $d$. In addition, it plays the role of the Gaussian for periodic variables, which makes this approach even more appealing [72].

We also observe that $\left|\psi_{0}\right\rangle$ satisfies a "parity" condition: if we write it as $\left|\psi_{0}\right\rangle=\sum_{\ell} c_{\ell}|\ell\rangle$, then $c_{\ell}=c_{-\ell}$. This guarantees that the average values of $\hat{U}$ and $\hat{V}$ in $\left|\psi_{0}\right\rangle$ are the same: $\left\langle\psi_{0}|\hat{U}| \psi_{0}\right\rangle=\left\langle\psi_{0}|\hat{V}| \psi_{0}\right\rangle$.

The coherent states (3.13) have properties fully analogous to the standard ones for continuous variables, as one can check with little effort.

The Harper Hamiltonian commutes with the Fourier operator $[\hat{\mathcal{F}}, \hat{H}]=0$. In fact, the state 3.16 is an approximate eigenstate of (3.14) in the high-dimensional limit

$$
\hat{H}\left|\psi_{0}\right\rangle \simeq\left(\frac{\pi}{d}-\frac{\pi^{2}}{2 d^{2}}+\frac{\pi^{3}}{6 d^{3}}\right)\left|\psi_{0}\right\rangle, \quad d \gg 1,
$$

which provides another argument for its use as a reference.

Finally, according to the recent results in Refs. [73] and [74], the following uncertainty relation holds

$$
(\Delta U)^{2}(\Delta V)^{2} \geq \frac{\pi^{2}}{d^{2}}
$$

where $(\Delta U)^{2}=1-|\langle\psi|\hat{U}| \psi\rangle|^{2}$ [and an analogous expression for $\left.(\Delta V)^{2}\right]$ denotes the circular dispersion, which is the natural generalization of variance for unitary operators. One can check that $\left|\psi_{0}\right\rangle$ saturates this inequality, confirming that it is also a minimum uncertainty state.

\section{Quasidistribution functions}

The displacement operators lead us to introduce a Hermitian $s$-ordered kernel

$$
\begin{aligned}
\hat{w}^{(s)}(m, n) & =\frac{1}{d} \sum_{k, l} \omega(n k-m l) \hat{D}(m, n) \\
& \times\left\langle\psi_{0}|\hat{D}(m, n)| \psi_{0}\right\rangle^{-s}
\end{aligned}
$$

which, as $\hat{w}^{(s)}(q, p)$ in Eq. 2.11, appears as a double Fourier transform of $\hat{D}$ with a weight determined by the operator ordering. However, here the parameter $s$ takes only discrete values $(s=-1,0,1)$.

These kernels are normalized and covariant under transformations of the generalized Pauli group

$$
\hat{D}(m, n) \hat{w}^{(s)}(k, l) \hat{D}^{\dagger}(m, n)=\hat{w}^{(s)}(k+m, l+n) .
$$




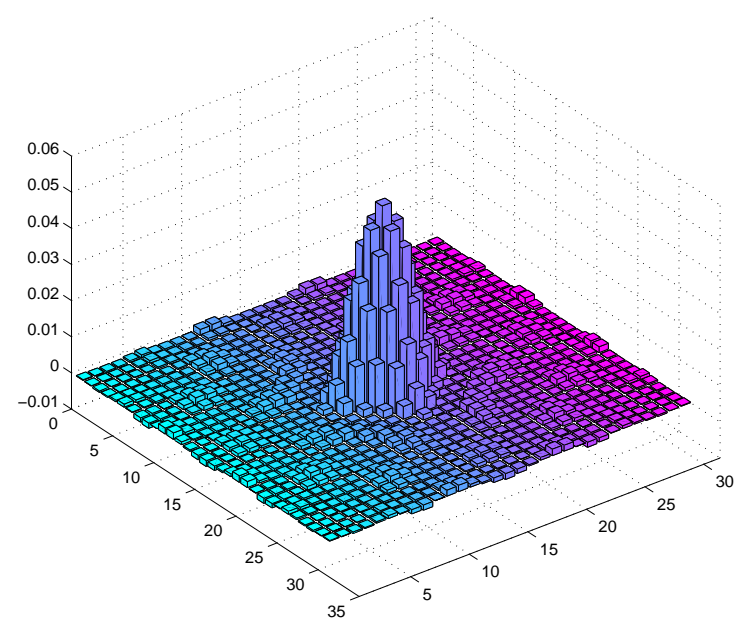

FIG. 1: $Q$ function 3.28 for the reference state $\left|\psi_{0}\right\rangle$, which plays the role of vacuum for continuous states.

They can be then conveniently represented as

$$
\hat{w}^{(s)}(m, n)=\hat{D}(m, n) \hat{w}^{(s)}(0,0) \hat{D}^{\dagger}(m, n),
$$

where, according to Eq. (3.12), $\hat{w}^{(s)}(0,0)$ coincides with the parity for $s=0(d \neq 2)$, as in the continuous case.

The $s$-ordered quasidistribution functions $W_{\hat{\varrho}}^{(s)}$ are generated through the mapping

$$
W_{\varrho}^{(s)}(m, n)=\operatorname{Tr}\left[\varrho \hat{~} \hat{w}^{(s)}(m, n)\right],
$$

which is invertible, so that

$$
\hat{\varrho}=\frac{1}{d} \sum_{m, n} \hat{w}^{-(s)}(m, n) W^{(s)}(m, n) .
$$

These functions fulfill all the basic properties required for the probabilistic description we are looking for. Let us apply them to the reference state $\left|\psi_{0}\right\rangle$ (notice that any other coherent state is just a displaced copy of this one). The corresponding Wigner function $(s=0)$ can be obtained after some algebra. We omit the details and merely quote the final result:

$$
\begin{aligned}
W_{\left|\psi_{0}\right\rangle}(m, n) & =\frac{d}{C} \sum_{k} \sum_{p, q \in \mathbb{Z}} \omega[(2 k-1-2 m) n] \\
& \times \exp [-k+2 m+q d-(d-1) / 2]^{2} \\
& \times \exp \left[-(\pi / d)(k+p d-d / 2)^{2}\right], \quad
\end{aligned}
$$

which, in the limit $d \gg 1$, can be approximated by the compact expression

$$
\begin{aligned}
W_{\left|\psi_{0}\right\rangle}^{(0)}(m, n) & \simeq \frac{\sqrt{2}}{d^{3 / 2}} \sum_{k, l}(-1)^{k l} \omega(m k-n l) \\
& \times \exp \left[-\pi\left(k^{2}+l^{2}\right) /(2 d)\right] .
\end{aligned}
$$

The $Q$ function $(s=-1)$ for the same state $\left|\psi_{0}\right\rangle$ reduces to

$$
Q_{\left|\psi_{0}\right\rangle}=\left|\left\langle\psi_{0}|\hat{D}(m, n)| \psi_{0}\right\rangle\right|^{2},
$$

which exhibits the additional interesting symmetry

$$
Q_{\left|\psi_{0}\right\rangle}(m, n)=Q_{\left|\psi_{0}\right\rangle}(-n, m) .
$$

In Fig.11 we have plotted this $Q$ function for a 31-dimensional system. The aspect of the figure confirms the issues one expects from a fairly localized Gaussian state.

\section{MANY QUDITS}

\section{A. Discrete phase space}

Next, we consider a system of $n$ identical qudits, living in the Hilbert space $\mathcal{H}_{d^{n}}$. Instead of natural numbers, it is convenient to use elements of the finite field $\mathrm{GF}\left(d^{n}\right)$ to label states: in this way we can almost directly translate all the properties studied before for a single qudit and we can endow the phasespace with many of the geometrical properties of the ordinary plane [53]. In the Appendix we briefly summarize the basic notions of finite fields needed to proceed.

We denote by $|\lambda\rangle$ [from here on, Greek letters will label elements in the field $\left.\mathrm{GF}\left(d^{n}\right)\right]$ an orthonormal basis in the Hilbert space of the system. Operationally, the elements of the basis can be labeled by powers of a primitive element using, for instance, the polynomial or the normal basis.

The generators of the Pauli group act now as

$$
\hat{U}_{\nu}|\lambda\rangle=|\lambda+\nu\rangle, \quad \hat{V}_{\mu}|\lambda\rangle=\chi(\mu \lambda)|\lambda\rangle,
$$

where $\chi(\lambda)$ is an additive character (defined in the Appendix) and the Weyl form of the commutation relations reads as

$$
\hat{V}_{\mu} \hat{U}_{\nu}=\chi(\mu \nu) \hat{U}_{\nu} \hat{V}_{\mu} .
$$

The finite Fourier transform [75]

$$
\hat{\mathcal{F}}=\frac{1}{\sqrt{d^{n}}} \sum_{\lambda, \lambda^{\prime}} \chi\left(\lambda \lambda^{\prime}\right)|\lambda\rangle\left\langle\lambda^{\prime}\right|
$$

allows us to introduce the conjugate basis $|\hat{\lambda}\rangle=\hat{\mathcal{F}}|\lambda\rangle$ and also we have

$$
\hat{V}_{\mu}=\hat{\mathcal{F}} \hat{U}_{\mu} \hat{\mathcal{F}}^{\dagger} .
$$

In this way, the concepts delineated in the previous section can be immediately generalized. For example, the displacement operators are

$$
\hat{D}(\mu, \nu)=\phi(\mu, \nu) \hat{U}_{\nu} \hat{V}_{\mu},
$$

where the phase $\phi(\mu, \nu)$ must satisfy the conditions

$$
\phi(\mu, \nu) \phi^{*}(\mu, \nu)=1, \quad \phi(\mu, \nu) \phi(-\mu,-\nu)=\chi(-\mu \nu),
$$

to guarantee the unitarity and orthogonality of $\hat{D}$. We also impose $\phi(\mu, 0)=1$ and $\phi(0, \nu)=1$, which means that the displacements along the "position" axis $\mu$ and the "momentum" axis $\nu$ are not associated with any phase. 
For fields of odd characteristics one possible form of this phase is

$$
\phi(\mu, \nu)=\chi\left(-2^{-1} \mu \nu\right),
$$

and we have then the same composition law as in Eq. (3.11), namely

$$
\begin{aligned}
\hat{D}\left(\mu_{1}, \nu_{1}\right) \hat{D}\left(\mu_{2}, \nu_{2}\right) & =\chi\left[2^{-1}\left(\mu_{1} \nu_{2}-\mu_{2} \nu_{1}\right)\right] \\
& \times \hat{D}\left(\mu_{1}+\mu_{2}, \nu_{1}+\nu_{2}\right) .
\end{aligned}
$$

\section{B. Coherent states}

Given our previous discussion, it seems reasonable to extend the coherent states $(3.13)$ in the form

$$
|\mu, \nu\rangle=\hat{D}(\mu, \nu)\left|\Psi_{0}\right\rangle,
$$

where $\left|\Psi_{0}\right\rangle$ is a reference state to be determined. In the continuous case, the extension of coherent states (2.6) to many degrees of freedom is straightforward: they are simply obtained by taking the direct product of single-mode coherent states. To reinterpret 4.9 in the same spirit, one needs first to map the abstract Hilbert space $\mathcal{H}_{d^{n}}$, where the $n$-qudit system lives, into $n$ single-qudit Hilbert spaces $\mathcal{H}_{d} \otimes \cdots \otimes \mathcal{H}_{d}$. This is achieved by expanding any field element in a convenient basis $\left\{\theta_{j}\right\}(j=1, \ldots, n)$, so that

$$
\lambda=\sum_{j} \ell_{j} \theta_{j}
$$

where $\ell_{j} \in \mathbb{Z}_{d}$. Then, we can represent the states as

$$
|\lambda\rangle=\left|\ell_{1}\right\rangle \otimes \cdots \otimes\left|\ell_{n}\right\rangle=\left|\ell_{1}, \ldots, \ell_{n}\right\rangle,
$$

and the coefficients $\ell_{j}$ play the role of quantum numbers for each qudit.

The use of the selfdual basis is especially advantageous, since only then the basic operators (and the Fourier operator) factorize in terms of single-qudit analogues

$$
\hat{U}_{\nu}=\hat{U}^{n_{1}} \otimes \cdots \otimes \hat{U}^{n_{n}}, \quad \hat{V}_{\mu}=\hat{V}^{m_{1}} \otimes \cdots \otimes \hat{V}^{m_{n}},
$$

and the displacement operators factorize accordingly

$$
\hat{D}(\mu, \nu)=\hat{D}\left(m_{1}, n_{1}\right) \otimes \cdots \otimes \hat{D}\left(m_{n}, n_{n}\right),
$$

where $m_{j}, n_{j} \in \mathbb{Z}_{d}$ are the coefficients of the expansion of $\mu$ and $\nu$ in the basis, respectively. In consequence, the eigenstates of the Fourier transform are direct product of singlequdit eigenstates and we can write for the reference state

$$
\left|\Psi_{0}\right\rangle=\bigotimes_{j=1}^{n}\left|\psi_{0 j}\right\rangle,
$$

where $\left|\psi_{0 j}\right\rangle$ are of the form (3.16) for each qudit (with $d>2$ ). For qubits, we have [76]

$$
\left|\Psi_{0}\right\rangle=\bigotimes_{j=1}^{n} \frac{(|0\rangle+\xi|1\rangle)_{j}}{\left(1+\xi^{2}\right)^{1 / 2}}
$$

with $\xi=\sqrt{2}-1$.

Unfortunately, the selfdual basis can be constructed only if either $d$ is even or both $n$ and $d$ are odd. This means that for such a simple system as two qutrits, this privileged basis does not exist. Nevertheless, one can always find an almost selfdual basis and one can proceed much along the same lines with minor modifications (the interested reader can consult the comprehensive review [51] for a full account of these methods).

It is interesting to stress that for $n$ qubits, the reference state (4.15) can be elegantly written in terms of the field elements $\mathrm{GF}\left(2^{n}\right)$ as follows

$$
\left|\Psi_{0}\right\rangle=\frac{1}{\left(1+\xi^{2}\right)^{n / 2}} \sum_{\alpha \in \mathrm{GF}\left(2^{n}\right)} \xi^{h(\alpha)}|\alpha\rangle,
$$

where the function $h(\alpha)$ counts the number of nonzero coefficients $a_{j}$ in the expansion of $\alpha$ in the basis.

The operator transforming from an arbitrary basis $\left\{\theta_{j}^{\prime}\right\}$ into the selfdual one $\left\{\theta_{j}\right\}$ is given by

$$
\hat{\mathcal{T}}=\sum_{\mu \in G F\left(2^{n}\right)}\left|m_{1}, \ldots, m_{n}\right\rangle\left\langle m_{1}^{\prime}, \ldots, m_{n}^{\prime}\right|,
$$

where

$$
\mu=\sum_{j} m_{j}^{\prime} \theta_{j}^{\prime}=\sum_{j} m_{j} \theta_{j}
$$

The operator $\hat{\mathcal{T}}$ is always a permutation and plays the role of an entangling (nonlocal) operator.

Let us examine the simple yet illustrative example of a twoqubit coherent state. According to Eq. 4.16, we have

$$
\left|\Psi_{0}\right\rangle=\frac{1}{1+\xi^{2}}\left(|0\rangle+\xi|\sigma\rangle+\xi\left|\sigma^{2}\right\rangle+\xi^{2}\left|\sigma^{3}\right\rangle\right)
$$

where $\sigma$ is a primitive element. The selfdual basis is $\left\{\sigma, \sigma^{2}\right\}$, and we have the representation

$$
\begin{gathered}
|0\rangle=|00\rangle=\left(\begin{array}{l}
0 \\
0 \\
0 \\
1
\end{array}\right), \quad|\sigma\rangle=|10\rangle=\left(\begin{array}{l}
0 \\
0 \\
1 \\
0
\end{array}\right) \\
\left|\sigma^{2}\right\rangle=|01\rangle=\left(\begin{array}{l}
0 \\
1 \\
0 \\
0
\end{array}\right), \quad\left|\sigma^{3}\right\rangle=|11\rangle=\left(\begin{array}{l}
1 \\
0 \\
0 \\
0
\end{array}\right)
\end{gathered}
$$

In consequence,

$$
\begin{aligned}
\left|\Psi_{0}\right\rangle & =\frac{1}{1+\xi^{2}}\left(\begin{array}{c}
\xi^{2} \\
\xi \\
\xi \\
1
\end{array}\right) \\
& =\frac{1}{\sqrt{1+\xi^{2}}}\left(\begin{array}{c}
\xi \\
1
\end{array}\right) \otimes \frac{1}{\sqrt{1+\xi^{2}}}\left(\begin{array}{l}
\xi \\
1
\end{array}\right)
\end{aligned}
$$


In a non selfdual basis, such as $\left\{\sigma, \sigma^{3}\right\}$, we have

$$
|0\rangle=|00\rangle, \quad|\sigma\rangle=|10\rangle, \quad\left|\sigma^{3}\right\rangle=|01\rangle, \quad\left|\sigma^{2}\right\rangle=|11\rangle,
$$

and

$$
\left|\Psi_{0}\right\rangle=\frac{1}{1+\xi^{2}}\left(\begin{array}{c}
\xi \\
\xi^{2} \\
\xi \\
1
\end{array}\right)
$$

The transition operator 4.17 turns out to be

$$
\hat{\mathcal{T}}=\left(\begin{array}{llll}
0 & 1 & 0 & 0 \\
1 & 0 & 0 & 0 \\
0 & 0 & 1 & 0 \\
0 & 0 & 0 & 1
\end{array}\right)
$$

which is nothing but a matrix representation of the CNOT operator.

\section{Quasidistribution functions}

The displacement operators 4.7 immediately suggest to introduce an $s$-ordered kernel

$$
\begin{aligned}
\hat{w}^{(s)}(\mu, \nu) & =\frac{1}{d^{n}} \sum_{\lambda, \kappa} \chi(\mu \lambda-\nu \kappa) \hat{D}(\mu, \nu) \\
& \times\left\langle\Psi_{0}|\hat{D}(\mu, \nu)| \Psi_{0}\right\rangle^{-s},
\end{aligned}
$$

which, in view of Eq. 4.3), can also be interpreted as a double Fourier transform of $\hat{D}(\mu, \nu)$. We can next introduce $s$ ordered quasidistribution functions through

$$
W_{\varrho}^{(s)}(\mu, \nu)=\operatorname{Tr}\left[\varrho \hat{\varrho} \hat{w}^{(s)}(\mu, \nu)\right],
$$

and the inversion relation reads as

$$
\hat{\varrho}=\frac{1}{d^{n}} \sum_{\mu, \nu} \hat{w}^{(-s)}(\mu, \nu) W_{\varrho}^{(s)}(\mu, \nu) .
$$

Due to the factorization of the character in the selfdual basis, the kernels $\hat{w}^{(s)}(\mu, \nu)$ factorize in this basis

$$
\hat{w}^{(s)}(\mu, \nu)=\prod_{j} \hat{w}^{(s)}\left(m_{j}, n_{j}\right)
$$

and, consequently, also do the corresponding quasidistributions

$$
W_{\hat{\varrho}}^{(s)}(\mu, \nu)=\prod_{j} W_{\hat{\varrho}_{j}}^{(s)}\left(m_{j}, n_{j}\right) .
$$

For the particular case of the Wigner function, one can check that

$$
\sum_{\mu, \nu} W_{\varrho}(\mu, \nu) \delta_{\nu, \alpha \mu+\beta}=\sum_{\mu, \nu} W_{\varrho}(\mu, \nu) \delta_{\nu,-\alpha^{-1} \mu-\beta},
$$

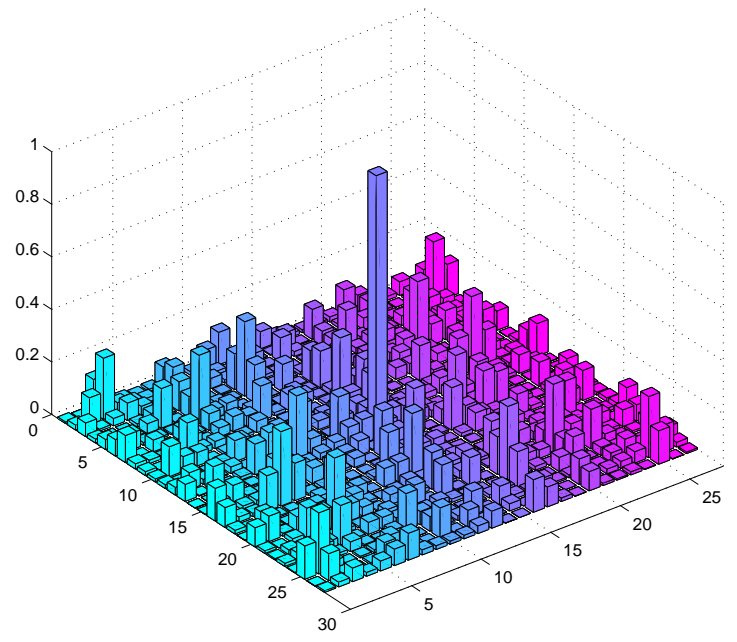

FIG. 2: $Q$ function for the reference state $\left|\Psi_{0}\right\rangle$, for a system of three qutrits. The order in the axes is as follows: $\sigma^{13}, \sigma^{17}, \sigma^{14}, \sigma, \sigma^{2}$, $\sigma^{21}, \sigma^{23}, \sigma^{7}, \sigma^{15}, \sigma^{4}, \sigma^{16}, \sigma^{6}, \sigma^{8}, 0, \sigma^{9}, \sigma^{12}, \sigma^{25}, \sigma^{24}, \sigma^{5}, \sigma^{3}$, $\sigma^{19}, \sigma^{11}, \sigma^{22}, \sigma^{10}, \sigma^{20}, \sigma^{18}, \sigma^{26}$, with $\sigma$ the primitive element.

that is, the sum over a line of slope $\alpha$ is the same as over a line of slope $-\alpha^{-1}$. The sum over the axes $\mu$ and $\nu$ are thus equal

$$
\sum_{\mu, \nu} W_{\hat{\varrho}}(\mu, \nu) \delta_{\nu, 0}=\sum_{\mu, \nu} W_{\hat{\varrho}}(\mu, \nu) \delta_{\mu, 0} .
$$

Note also, that the $Q$ function reduces to

$$
Q_{\hat{\varrho}}(\mu, \nu)=\langle\mu, \nu|\hat{\varrho}| \mu, \nu\rangle \text {. }
$$

In Fig. 2 we have plotted this $Q$ function for the reference state $\left|\Psi_{0}\right\rangle$ in a system of three qutrits. The selfdual basis here is $\left\{\sigma, \sigma^{3}, \sigma^{9}\right\}$ and the primitive element is a solution of the irreducible polynomial $x^{3}+2 x^{2}+1=0$.

\section{SQUEEZED STATES}

Squeezed states constitute a simple nontrivial enlargement of the notion of coherent states. In continuous variables, a squeezed state is a minimum uncertainty state that my have less fluctuations in one quadrature $(\hat{q}$ or $\hat{p})$ than a coherent state. They are generated from the vacuum by using the unitary squeeze operator

$$
\hat{S}(\mathfrak{s})=\exp [-i \mathfrak{s}(\hat{q} \hat{p}+\hat{p} \hat{q})]
$$

with a subsequent displacement to an arbitrary point in the complex plane

$$
|q, p ; \mathfrak{s}\rangle=\hat{D}(q, p) \hat{S}(\mathfrak{s})\left|\psi_{0}\right\rangle .
$$

It is easy to check that

$$
\hat{S}(\mathfrak{s}) \hat{q} \hat{S}^{\dagger}(\mathfrak{s})=\hat{q} e^{\mathfrak{s}}, \quad \hat{S}(\mathfrak{s}) \hat{p} \hat{S}^{\dagger}(\mathfrak{s})=\hat{p} e^{-\mathfrak{s}}
$$

so that, the operator $\hat{S}(\mathfrak{s})$ attenuates one quadrature and amplifies the canonical one by the same factor determined by the 
squeeze factor $\mathfrak{s}$, which, for simplicity, we have taken as real. As a simple consequence of (5.3) one can verify the transformations for $\hat{U}(q)$ and $\hat{V}(p)$ :

$$
\hat{S}(\mathfrak{s}) \hat{U}(q) \hat{S}^{\dagger}(\mathfrak{s})=U^{\mathfrak{s}}(q), \quad \hat{S}(\mathfrak{s}) \hat{V}(p) \hat{S}^{\dagger}(\mathfrak{s})=\hat{V}^{-\mathfrak{s}}(p) .
$$

For a single qudit, squeezed states have been recently considered in detail in Ref. [61], using an extended CahillGlauber formalism. Here, we prefer to follow an alternative approach and define a squeeze operator as

$$
\hat{S}_{s}=\sum_{\ell}|\ell\rangle\langle s \ell|, \quad s \in \mathbb{Z}_{d} .
$$

At first sight, this can appear as a rather abstract choice. However, notice that

$$
\hat{S}_{s}^{\dagger} \hat{U}^{n} \hat{S}_{s}^{\dagger}=\hat{U}^{n s}, \quad \hat{S}_{s} \hat{V}^{m} \hat{S}_{s}=\hat{V}^{m s^{-1}}
$$

which is a direct translation of the action (5.4) to this discrete case. This also means that in the squeezed "vacuum"

$$
\left|\psi_{0} ; s\right\rangle=\hat{S}_{s}\left|\psi_{0}\right\rangle
$$

the average values of some powers of the displacement operators are the same

$$
\left\langle\psi_{0} ; s|\hat{U}| \psi_{0} ; s\right\rangle=\left\langle\psi_{0} ; s\left|\hat{V}^{s^{2}}\right| \psi_{0} ; s\right\rangle
$$

Perhaps, the clearest way to visualize this squeezing is to use a quasidistribution, such as, e.g., the Wigner function. If $\hat{\varrho}_{s}=\hat{S}_{s} \hat{\varrho} \hat{S}_{s}^{\dagger}$ denotes the density operator of a squeezed state, we have

$$
W_{\hat{\varrho}_{s}}(m, n)=W_{\hat{\varrho}}\left(s m, s^{-1} n\right),
$$

whose geometrical interpretation is obvious and is the phasespace counterpart of the property (5.3). For reasons that will become evident soon, we refer to this as "geometrical squeezing". We also note the following symmetry property of the Wigner function

$$
\sum_{m, n} W_{\hat{\varrho}_{s}}(m, n) \delta_{n, 0}=\sum_{m, n} W_{\hat{\varrho}_{s}-1}(m, n) \delta_{m, 0} \cdot
$$

For many qudits, our developed intuition suggests a direct translation of 5.5) in terms of the field elements in $\operatorname{GF}\left(d^{n}\right)$, namely

$$
\hat{S}_{\varsigma}=\sum_{\lambda}|\lambda\rangle\langle\varsigma \lambda|, \quad \varsigma \in \mathrm{GF}\left(d^{n}\right),
$$

in terms of which we can write relations similar to Eqs.(5.6)(5.9). In fact, one can define a squeezed "vacumm" as in Eq. (5.7), i.e., $\left|\Psi_{0} ; \varsigma\right\rangle=\hat{S}_{\varsigma}\left|\Psi_{0}\right\rangle$. In Fig. 3 we plot the Wigner function for this squeezed state in a system of three qutrits with $\varsigma=\sigma^{7}$.

Nevertheless, now the squeezing acquires a new physical perspective: the squeeze operator 5.11) cannot be, in general, factorized into a product of single qudit squeezing operators. This means that by applying $\hat{S}_{\varsigma}$ to a factorized state

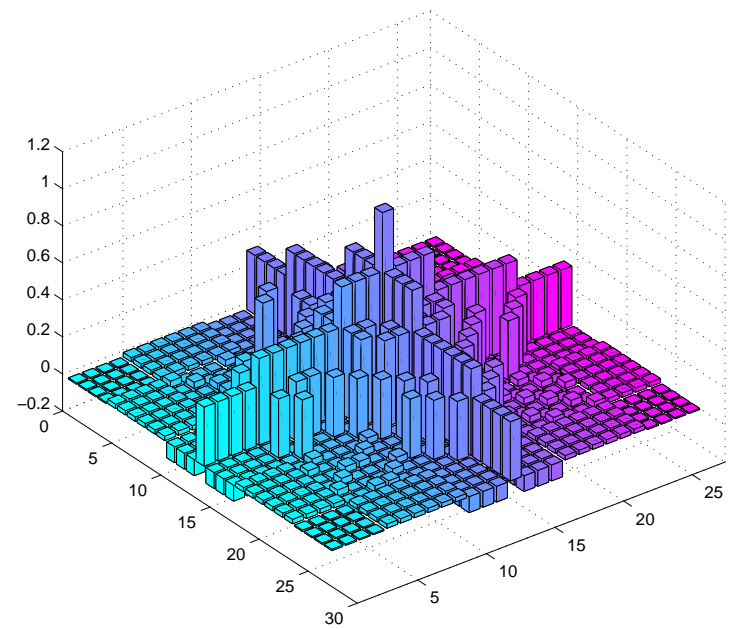

FIG. 3: Wigner function for a squeezed "vacuum" state $\left|\Psi_{0}, \varsigma\right\rangle$, for a system of three qutrits, with the same order in the axes as in Fig. 2.

we generate correlations between qudits; i.e., we create entangled states. The most striking example is of course the $n$ qubit case, since there is no single qubit squeezing.

To understand these correlations consider a general factorized state

$$
|\Psi\rangle=\sum_{\lambda} C_{\lambda}|\lambda\rangle=\sum_{c_{\ell_{1}}, \ldots, c_{\ell_{n}}} c_{\ell_{1}} \ldots c_{\ell_{n}}\left|\ell_{1}, \ldots, \ell_{n}\right\rangle,
$$

and apply (5.11). The resulting state turns out to be

$$
\hat{S}_{\varsigma}|\Psi\rangle=\sum_{\ell_{1}, \cdots, \ell_{n}} C_{m_{1} \theta_{1}+\ldots+m_{n} \theta_{n}}\left|\ell_{1}, \ldots, \ell_{n}\right\rangle,
$$

where

$$
\begin{gathered}
m_{i}=\sum_{j, k=0}^{d-1} f_{i j k} \ell_{j} h_{k}, \\
f_{i j k}=\operatorname{tr}\left(\theta_{i} \theta_{j} \theta_{k}\right), \quad h_{k}=\operatorname{tr}\left(\varsigma^{-1} \theta_{k}\right),
\end{gathered}
$$

$\operatorname{tr}$ (written in lower case) is the trace operation in the field (see the Appendix) and $\left\{\theta_{j}\right\}$ is the basis.

As a example let us consider a three-qubit system. Now the selfdual basis is $\left\{\theta_{1}=\sigma^{3}, \theta_{2}=\sigma^{5}, \theta_{3}=\sigma^{6}\right\}$, where $\sigma$ is a primitive element, solution of the irreducible polynomial $x^{3}+x+1=0$. The result of applying $\hat{S}_{\sigma^{k}}$ to the state 5.12 can be expressed in terms of

$$
\begin{aligned}
\hat{S}_{\sigma}|\Psi\rangle & =\sum_{\lambda \in \mathrm{GF}\left(2^{3}\right)} C_{\sigma^{6} \lambda}|\lambda\rangle=\sum_{p, q, r \in \mathbb{Z}_{2}} c_{p+q} c_{p+r} c_{q}|p, q, r\rangle, \\
\hat{S}_{\sigma^{3}}|\Psi\rangle & =\sum_{\lambda \in \mathrm{GF}\left(2^{3}\right)} C_{\sigma^{4} \lambda}|\lambda\rangle=\sum_{p, q, r \in \mathbb{Z}_{2}} c_{p+q+r} c_{p+r} c_{r}|p, q, r\rangle .
\end{aligned}
$$

In fact, the transformations $\left\{\hat{S}_{\sigma^{5}}, \hat{S}_{\sigma^{6}}\right\}$ generate the same entanglement (except for permutations) as $\hat{S}_{\sigma^{3}}$, while $\left\{\hat{S}_{\theta^{2}}, \hat{S}_{\theta^{4}}\right\}$ generate the same entanglement (again except for permutations) as $\hat{S}_{\sigma}$. 


\section{CONCLUDING REMARKS}

In summary, we have provided a handy toolbox for dealing with many-qudit systems in phase space. The mathematical basis of our approach is the use algebraic field extensions that produce results in composite dimensions in a manner very close to the continuous case.

Another major advantage of our theory relies on the use of the finite Fourier transform and its eigenstates for the definition of coherent states. We believe that this makes a clear connection with the standard coherent states for continuous variables and constitutes an elegant solution to this problem. The factorization properties of the resulting coherent states in different bases is also an interesting question.

We have also established a set of important results that have allowed us to obtain discrete analogs of squeezed states. While for a single qudit, these squeezed states have the properties one would expect from our continuous-variable experience, for many qudits an amazing relation with entanglement appears.

We think that the techniques presented here are more than a mere academic curiosity, for they are immediately applicable to a variety of experiments involving qudit systems.

\section{APPENDIX A: FINITE FIELDS}

In this appendix we briefly recall the minimum background needed in this paper. The reader interested in more mathematical details is referred, e.g., to the excellent monograph by Lidl and Niederreiter [35].

A commutative ring is a nonempty set $R$ furnished with two binary operations, called addition and multiplication, such that it is an Abelian group with respect the addition, and the multiplication is associative. Perhaps, the motivating example is the ring of integers $\mathbb{Z}$, with the standard sum and multiplication. On the other hand, the simplest example of a finite ring is the set $\mathbb{Z}_{n}$ of integers modulo $n$, which has exactly $n$ elements.

A field $F$ is a commutative ring with division, that is, such that 0 does not equal 1 and all elements of $F$ except 0 have a multiplicative inverse (note that 0 and 1 here stand for the identity elements for the addition and multiplication, respectively, which may differ from the familiar real numbers 0 and 1). Elements of a field form Abelian groups with respect to addition and multiplication (in this latter case, the zero element is excluded).

The characteristic of a finite field is the smallest integer $d$ such that

$$
d 1=\underbrace{1+1+\ldots+1}_{d \text { times }}=0
$$

and it is always a prime number. Any finite field contains a prime subfield $\mathbb{Z}_{d}$ and has $d^{n}$ elements, where $n$ is a natural number. Moreover, the finite field containing $d^{n}$ elements is unique and is called the Galois field $\operatorname{GF}\left(d^{n}\right)$.

Let us denote as $\mathbb{Z}_{d}[x]$ the ring of polynomials with coefficients in $\mathbb{Z}_{d}$. Let $P(x)$ be an irreducible polynomial of degree $n$ (i.e., one that cannot be factorized over $\mathbb{Z}_{d}$ ). Then, the quotient space $\mathbb{Z}_{d}[X] / P(x)$ provides an adequate representation of $\mathrm{GF}\left(d^{n}\right)$. Its elements can be written as polynomials that are defined modulo the irreducible polynomial $P(x)$. The multiplicative group of $\mathrm{GF}\left(d^{n}\right)$ is cyclic and its generator is called a primitive element of the field.

As a simple example of a nonprime field, we consider the polynomial $x^{2}+x+1=0$, which is irreducible in $\mathbb{Z}_{2}$. If $\sigma$ is a root of this polynomial, the elements $\left\{0,1, \sigma, \sigma^{2}=\right.$ $\left.\sigma+1=\sigma^{-1}\right\}$ form the finite field $\operatorname{GF}\left(2^{2}\right)$ and $\sigma$ is a primitive element.

A basic map is the trace

$$
\operatorname{tr}(\lambda)=\lambda+\lambda^{2}+\ldots+\lambda^{d^{n-1}} .
$$

It is always in the prime field $\mathbb{Z}_{d}$ and satisfies

$$
\operatorname{tr}\left(\lambda+\lambda^{\prime}\right)=\operatorname{tr}(\lambda)+\operatorname{tr}\left(\lambda^{\prime}\right) .
$$

In terms of it we define the additive characters as

$$
\chi(\lambda)=\exp \left[\frac{2 \pi i}{p} \operatorname{tr}(\lambda)\right]
$$

which posses two important properties:

$$
\chi\left(\lambda+\lambda^{\prime}\right)=\chi(\lambda) \chi\left(\lambda^{\prime}\right), \quad \sum_{\lambda^{\prime} \in \mathrm{GF}\left(d^{n}\right)} \chi\left(\lambda \lambda^{\prime}\right)=d^{n} \delta_{0, \lambda}
$$

Any finite field $\mathrm{GF}\left(d^{n}\right)$ can be also considered as an $n$ dimensional linear vector space. Given a basis $\left\{\theta_{j}\right\},(j=$ $1, \ldots, n)$ in this vector space, any field element can be represented as

$$
\lambda=\sum_{j=1}^{n} \ell_{j} \theta_{j}
$$

with $\ell_{j} \in \mathbb{Z}_{d}$. In this way, we map each element of $\operatorname{GF}\left(d^{n}\right)$ onto an ordered set of natural numbers $\lambda \Leftrightarrow\left(\ell_{1}, \ldots, \ell_{n}\right)$.

Two bases $\left\{\theta_{1}, \ldots, \theta_{n}\right\}$ and $\left\{\theta_{1}^{\prime}, \ldots, \theta_{n}^{\prime}\right\}$ are dual when

$$
\operatorname{tr}\left(\theta_{k} \theta_{l}^{\prime}\right)=\delta_{k, l}
$$

A basis that is dual to itself is called selfdual.

There are several natural bases in $\operatorname{GF}\left(d^{n}\right)$. One is the polynomial basis, defined as

$$
\left\{1, \sigma, \sigma^{2}, \ldots, \sigma^{n-1}\right\}
$$

where $\sigma$ is a primitive element. An alternative is the normal basis, constituted of

$$
\left\{\sigma, \sigma^{d}, \ldots, \sigma^{d^{n-1}}\right\}
$$

The choice of the appropriate basis depends on the specific problem at hand. For example, in $\mathrm{GF}\left(2^{2}\right)$ the elements $\left\{\sigma, \sigma^{2}\right\}$ are both roots of the irreducible polynomial. The polynomial basis is $\{1, \sigma\}$ and its dual is $\left\{\sigma^{2}, 1\right\}$, while the normal basis $\left\{\sigma, \sigma^{2}\right\}$ is selfdual.

The selfdual basis exists if and only if either $d$ is even or both $n$ and $d$ are odd. However for every prime power $d^{n}$, 
there exists an almost selfdual basis of $\operatorname{GF}\left(d^{n}\right)$, which satisfies the properties: $\operatorname{tr}\left(\theta_{i} \theta_{j}\right)=0$ when $i \neq j$ and $\operatorname{tr}\left(\theta_{i}^{2}\right)=1$, with one possible exception. For instance, in the case of two qutrits $\mathrm{GF}\left(3^{2}\right)$, a selfdual basis does not exist and two elements $\left\{\sigma^{2}, \sigma^{4}\right\}, \sigma$ being a root of the irreducible polynomial $x^{2}+x+2=0$, form an almost selfdual basis

$$
\operatorname{tr}\left(\sigma^{2} \sigma^{2}\right)=1, \quad \operatorname{tr}\left(\sigma^{4} \sigma^{4}\right)=2, \quad \operatorname{tr}\left(\sigma^{2} \sigma^{4}\right)=0 .
$$

[1] H. Weyl, Gruppentheorie und Quantemechanik (Hirzel-Verlag, Leipzig, 1928).

[2] E. P. Wigner, Phys. Rev. 40, 749 (1932).

[3] J. E. Moyal, Proc. Cambridge Phil. Soc. 45, 99 (1949).

[4] H.-W. Lee, Phys. Rep. 259, 147 (1995).

[5] F. E. Schroek, Quantum Mechanics on Phase Space (Kluwer, Dordrecht, 1996).

[6] W. P. Schleich, Quantum Optics in Phase Space (Wiley-VCH, Berlin, 2001).

[7] C. K. Zachos, D. B. Fairlie, and T. L. Curtright, eds., Quantum mechanics in phase space (World Scientific, Singapore, 2005).

[8] G. Kimura, Phys. Lett. A 314, 339 (2003).

[9] S. G. Schirmer, T. Zhang, and J. V. Leahy, J. Phys. A 37, 1389 (2004).

[10] B. Kostant, Lect. Notes Math. 170, 87 (1970).

[11] A. A. Kirillov, Elements of the Theory of Representations (Springer-Verlag, Berlin, 1976.).

[12] J. H. Hannay and M. V. Berry, Physica D 1, 267 (1980).

[13] U. Leonhardt, Phys. Rev. Lett. 74, 4101 (1995).

[14] C. Miquel, J. P. Paz, M. Saraceno, E. Knill, R. Laflamme, and C. Negrevergne, Nature (London) 418, 59 (2002).

[15] J. Schwinger, Proc. Natl. Acad. Sci. USA 46, 570 (1960).

[16] J. Schwinger, Prod. Natl. Acad. Sci. USA 46, 883 (1960).

[17] J. Schwinger, Proc. Natl. Acad. Sci. USA 46, 1401 (1960).

[18] F. A. Buot, Phys. Rev. B 10, 3700 (1973).

[19] O. Cohendet, P. Combe, M. Sirugue, and M. Sirugue-Collin, J. Phys. A 21, 2875 (1988).

[20] P. Kasperkovitz and M. Peev, Ann. Phys. 230, 21 (1994).

[21] T. Opatrný, V. Bužek, J. Bajer, and G. Drobný, Phys. Rev. A 52, 2419 (1995).

[22] A. M. F. Rivas and A. M. O. de Almeida, Ann. Phys. 276, 223 (1999).

[23] N. Mukunda, S. Chaturvedi, and R. Simon, J. Math. Phys. 45, 114 (2004).

[24] S. Chaturvedi, E. Ercolessi, G. Marmo, G. Morandi, N. Mukunda, and R. Simon, J. Phys. A 39, 1405 (2006).

[25] W. K. Wootters, Found. Phys. 16, 391 (1986).

[26] W. K. Wootters and B. D. Fields, Ann. Phys. 191, 363 (1989).

[27] W. K. Wootters, Found. Phys. 36, 112 (2006).

[28] W. K. Wootters and D. M. Sussman, arXiv:0704.1277 (2007).

[29] D. Galetti and A. F. R. de Toledo Piza, Physica A 149, 267 (1988).

[30] D. Galetti and A. F. R. de Toledo Piza, Physica A 186, 513 (1992).

[31] D. Galetti and A. de Toledo Piza, Physica A 214, 207 (1995).

[32] A. B. Klimov, L. L. Sánchez-Soto, and H. de Guise, J. Phys. A 38, 2747 (2005).

[33] A. B. Klimov, J. L. Romero, G. Björk, and L. L. Sánchez-Soto, J. Phys. A 40, 3987 (2007).

[34] A. B. Klimov, J. L. Romero, G. Björk, and L. L. Sánchez-Soto, Ann. Phys. 324, 53 (2009).

[35] R. Lidl and H. Niederreiter, Introduction to Finite Fields and their Applications (Cambridge University Press, Cambridge, 1986).
[36] R. J. Glauber, Phys. Rev. 131, 2766 (1963).

[37] E. C. G. Sudarshan, Phys. Rev. Lett. 10, 277 (1963).

[38] K. Husimi, Proc. Phys. Math. Soc. Japan 22, 264 (1940).

[39] M. Hillery, R. F. O'Connell, M. O. Scully, and E. P. Wigner, Phys. Rep. 106, 121 (1984).

[40] K. E. Cahill and R. J. Glauber, Phys. Rev. 177, 1857 (1969).

[41] R. L. Stratonovich, JETP 31, 1012 (1956).

[42] F. A. Berezin, Commun. Math. Phys. 40, 153 (1975).

[43] G. S. Agarwal, Phys. Rev. A 24, 2889 (1981).

[44] M. O. Scully, Phys. Rev. D 28, 2477 (1983).

[45] L. Cohen and M. O. Scully, Found. Phys. 16, 295 (1986).

[46] J. C. Varilly and J. M. Gracia-Bondía, Ann. Phys. 190, 107 (1989).

[47] S. Heiss and S. Weigert, Phys. Rev. A 63, 012105 (2000).

[48] J. P. Dowling, G. S. Agarwal, and W. P. Schleich, Phys. Rev. A 49, 4101 (1994).

[49] M. G. Benedict and A. Czirják, Phys. Rev. A 60, 4034 (1999).

[50] C. Brif and A. Mann, J. Phys. A 31, L9 (1998).

[51] G. Björk, A. B. Klimov, and L. L. Sánchez-Soto, Prog. Opt. 51, 469 (2008).

[52] W. K. Wootters, Ann. Phys. 176, 1 (1987).

[53] K. S. Gibbons, M. J. Hoffman, and W. K. Wootters, Phys. Rev. A 70, 062101 (2004).

[54] W. K. Wootters, IBM J. Res. Dev. 48, 99 (2004).

[55] M. Ruzzi, M. A. Marchiolli, and D. Galetti, J. Phys. A pp. 6239-6251 (2005).

[56] M. A. Marchiolli, M. Ruzzi, and D. B. F. Galetti, Phys. Rev. A 72, 042308 (2005).

[57] J. R. Klauder and B. S. Skagerstam, Coherent States: Applications in Physics and Mathematical Physics (World Scientific Publishing, Singapore, 1999).

[58] A. Perelomov, Generalized Coherent States and their Applications (Springer, Berlin, 1986).

[59] M. Ruzzi, J. Math. Phys 47, 063507 (2006).

[60] D. Galetti and M. A. Marchiolli, Ann. Phys. 249, 454 (1996).

[61] M. A. Marchiolli, M. Ruzzi, and D. Galetti, Phys. Rev. A 76, 032102 (2007).

[62] C. R. Putnam, Commutation Properties of Hilbert Space Operators and Related Topics (Springer, Berlin, 1967).

[63] A. Peres, Quantum Theory: Concepts and Methods (Kluwer, Dordrecht, 1993).

[64] A. Vourdas, Rep. Prog. Phys. 67, 267 (2004).

[65] I. Chuang and M. Nielsen, Quantum Computation and Quantum Information (Cambridge University Press, Cambridge, 2000).

[66] A. Vourdas, J. Phys. A 38, 8453 (2005).

[67] M. Saraceno, Ann. Phys. 199, 37 (1990).

[68] J. P. Paz, A. J. Roncaglia, and M. Saraceno, Phys. Rev. A 69, 032312 (2004).

[69] P. G. Harper, Proc. Phys. Soc. A 68, 874 (1955).

[70] M. L. Mehta, J. Math. Phys. 28, 781 (1987).

[71] D. Mumford, Tata Lectures on Theta I (Birkhauser, Boston, 1983).

[72] J. Řeháček, Z. Bouchal, R. Čelechovský, Z. Hradil, and L. L. 
Sánchez-Soto, Phys. Rev. A 77, 032110 (2008).

[73] G. W. Forbes, M. A. Alonso, and A. E. Siegman, J. Phys. A 36, 7027 (2003).

[74] S. Massar and P. Spindel, Phys. Rev. Lett. 100, 190401 (2008).
[75] A. Vourdas, J. Phys. A 40, R285 (2007).

[76] C. Muñoz, A. B. Klimov, L. L. Sánchez-Soto, and G. Björk, Int. J. Quantum Inf. 7, 17 (2009). 